\title{
Evaluation of Commercial Solid-Phase Extraction (SPE) Carrier Materials for the Selective Automated Enrichment of Monoterpenoides and their Analysis in Cough Drops, Mouthwashes and Bath Additives by Gas- Chromatography Mass Spectrometry (GC-MS)
}

\author{
C. Uhlschmied ${ }^{1}$, C. Krieg ${ }^{1}$, G. Abel ${ }^{2}$, M. Popp ${ }^{2}$, C.W. Huck ${ }^{*}, 1$ and G.K. Bonn ${ }^{1}$ \\ ${ }^{1}$ Institute of Analytical Chemistry and Radiochemistry, CCB-Center for Chemistry and Biomedicine, Leopold-Franzens \\ University, Innrain 80-82, 6020 Innsbruck, Austria \\ ${ }^{2}$ Bionorica SE, Kerschensteinerstr. 11-18, 92318 Neumarkt/Oberpfalz, Germany
}

\begin{abstract}
For sample preparation solid-phase extraction (SPE) became very popular during the 1980s and 1990s. Because method development and its optimization is very time consuming, in most cases $\mathrm{C}_{18}$ is used as standard carrier material without knowing if it possesses the highest efficiency. Therefore detailed studies with different stationary phases are necessary for optimal recovery rates. Due to the resulting high number of samples, automation is of crucial importance. In the study described, the extraction of five monoterpenoids (myrcene, citronellal, p-cymene, menthol and thymol) out of an ethanolic solution was optimized using automated SPE. An automated robotic system, the MEA Personal Purification System $^{\circledR}$ from PhyNexus ${ }^{\text {TM }}$ Inc. (http://www.phynexus.com/), was adopted for high-throughput SPE. It allows the simultaneous automatic extraction of 12 samples in parallel employing different carrier materials. Additionally, critical parameters of automation (speed, volume uptake, skipping of drying step) were variegated to obtain optimal recovery rates. Furthermore, extraction efficiency was compared with manual handling to evaluate its performance. The application of 18 different SPE carrier materials, $\mathrm{C}_{18}$ Hydra, $\mathrm{C}_{18}$ ec, $\mathrm{C}_{8}, \mathrm{C}_{4}, \mathrm{C}_{2}, \mathrm{C}_{6} \mathrm{H}_{5}, \mathrm{C}_{6} \mathrm{H}_{11}$ ec, $\mathrm{NO}_{2}, \mathrm{CN}_{2} \mathrm{NH}_{2} / \mathrm{C}_{18}$, Diol, HR-P, EASY, PA, Florisil ${ }^{\circledR}$, Davisil $^{\circledR}$, Strata- $^{\circledR}$ and cellulose pointed out huge differences in extraction efficiency. Finally, the optimized automated procedure was applied for the enrichment of monoterpenoides and their analysis in selected real samples including cough drops, mouthwash and bath additives by gas-chromatography mass spectrometry (GC-MS).
\end{abstract}

Keywords: Solid-Phase extraction, monoterpenoides, automation, gas-chromatography mass spectrometry, real sample.

\section{INTRODUCTION}

Solid-phase extraction (SPE) is one of the most important sample preparation techniques [1]. Its popularity increased with the need to decrease organic solvent usage [2]. It can be used for different tasks, also in combination: trace enrichment (concentration), matrix simplification (sample clean-up) and medium exchange (transfer from the sample matrix to a different solvent or to the gas phase) [3]. One can differentiate between cartridge based SPE and dispersive SPE. In dispersive SPE, the stationary phase is added to the liquid sample, mixed and finally filtered to remove the liquid phase or parts of the liquid phase are used for further analysis. This technique is very popular for the cleanup of e.g. pesticide samples [4]. There it is part of the so called QuEChERS method (Quick, Easy, Cheap, Effective, Rugged and Safe) [5].

In the beginnings of SPE $\mathrm{C}_{18}$-silicas differing only in particle size from those in high-performance liquid chromatography (HPLC) were used [2]. Nowadays sorbents especially designed for SPE are available, based on silica or

*Address correspondence to this author at the Institute of Analytical Chemistry and Radiochemistry, CCB-Center for Chemistry and Biomedicine, Leopold-Franzens University, Innrain 80-82, 6020 Innsbruck, Austria; Tel: +4351250757304; E-mail: Christian.W.Huck@uibk.ac.at on polymeric beads with a broad variety of functionalities [6]. The most common SPE materials are $\mathrm{C}_{18}$-silicas, but there are a wide range of silica sorbents with varying alkyl chain length available, e.g. $\mathrm{C}_{2}, \mathrm{C}_{4}, \mathrm{C}_{8}$. These materials show in most cases lower retention, but have the advantage to retain less interference. There are also phenyl and cyclohexyl phases available providing higher retention for some aromatic compounds [2]. To achieve high recovery rates, a large surface area is of great importance as the extraction efficiency depends on the equilibrium between the two phases. Typically $\mathrm{C}_{18}$-silica materials are made from silica with specific surface areas in the range of 500 to $600 \mathrm{~m}^{2} \mathrm{~g}^{-1}$, while certain polymeric resins designed for SPE reach high specific surface areas in the range of 700 to $1200 \mathrm{~m}^{2} \mathrm{~g}^{-1}$ (e.g. Chromabond ${ }^{\circledR}$ HR-P $1200 \mathrm{~m}^{2} \mathrm{~g}^{-1}$, Oasis ${ }^{\circledR}$ HLB $800 \mathrm{~m}^{2} \mathrm{~g}^{-1}$ ) and thus higher capacities $[2,3,7]$.

Another advantage of polymeric stationary phases is the better $\mathrm{pH}$ stability, while silica-based sorbents are only stable in a pH range of 2 to $8[3,8]$. Sun and Fritz found that extraction with polymeric resins resulted in higher recovery for many types of analytes $[9,6]$. Both silica and polymeric stationary phases have some disadvantages [10]. Due to its polar silanolic groups, silica is hydrophilic but is getting hydrophobic by the immobilized carbon chains. Polystyrene based materials have a hydrophobic character, too, resulting in poor surface contact under aqueous conditions $[1,6,11]$. 
To overcome this disadvantage, the material has to be exposed to an activating solvent, in most cases methanol. Low recovery rates can also be caused in case the column runs dry and air is introduced [2, 12]. The new hydrophilic lipophilic based materials, like Oasis ${ }^{\circledR}$ HLB, a macroporous poly(divinylbenzene-co-N-vinylpyrrolidone) polymer (Waters, Eschborn, Germany), or Strata- $X^{\circledR}$ (Phenomenex, Aschaffenburg, Germany), are water-wettable and presented as "universal" sorbent for acidic, basic and neutral analytes whether polar or unpolar. In addition to the hydrophobic interaction and the $\pi$ - $\pi$-interactions of the divinylbenzene, the pyrrolidone groups acts as a hydrogen acceptor $[2,3]$.

Manual SPE procedure is time consuming and a lot of mistakes can be made. The basic equipment necessary for manual SPE is the SPE cartridge, a syringe and a special adapter. This way the necessary pressure is applied. Using this equipment only one sample can be processed at a time. To increase the amount of sample processed at a time, vacuum manifolds for 6, 12 or 24 samples are available. For high sample throughput especially in routine analysis there are automated systems on the market. Extraction plate systems are able to handle 96 samples at a time. The SPE material is embedded in every well. The waste well plate is replaced after running the sample through the system by a collection well plate and the analytes are eluted. This system has a big disadvantage, the restriction in choice of SPE materials as the material is the same in each well and cannot be chosen separately [13]. PhyNexus Inc. developed a Personal Purification and Enrichment System, MEA ${ }^{\mathrm{TM}}$. The MEA $^{\mathrm{TM}}$ robot offers automated SPE for the simultaneous handling of up to 12 samples, while the sorbent can be chosen separately for each sample. Therefore the main application of the system lies in method development, optimization and medium throughput screening [13]. In case of really high sample numbers, Tecan ${ }^{\circledR}$ and Hamilton ${ }^{\circledR}$ offer fully automated SPE systems using 96 deep well plates for industrial and clinical applications.

The aim of the present study was to evaluate commercial available SPE materials for the selective automated enrichment of monoterpenoides and their analysis in cough drops, mouthwashes and bath additives by gaschromatography mass spectrometry (GC-MS)

\section{EXPERIMENTAL}

\subsection{Chemicals}

Methanol (gradient grade) was purchased from SigmaAldrich (Steinheim, Germany) and water was provided by the in-house water treatment plant (Barnstead Nanopure Infinity, Thermo Fisher Scientific, Dreieich, Germany).

\subsection{Samples}

Standards were purchased from Sigma-Aldrich (Steinheim, Germany). The mixed standard solutions contained alpha-pinene (98\%), beta-pinene (99\%), myrcene (>90\%), citronellal (purum), L-menthol (purum), p-cymene (99\%), borneol (puriss, p.a.) and thymol (99.5\%). High concentrated standard solution contained $500 \mathrm{mg} \mathrm{L}^{-1}$ alphapinene, $570 \mathrm{mg} \mathrm{L}^{-1}$ beta-pinene, $591 \mathrm{mg} \mathrm{L}^{-1}$ myrcene, $502 \mathrm{mg} \mathrm{L}^{-1}$ p-cymene, $543 \mathrm{mg} \mathrm{L}^{-1}$ citronellal, $740 \mathrm{mg} \mathrm{L}^{-1} \mathrm{~L}-$ menthol, $730 \mathrm{mg} \mathrm{L}^{-1}$ borneol and $485 \mathrm{mg} \mathrm{L}^{-1}$ thymol in methanol. Low concentrated standard solution contained
$123 \mathrm{mg} \mathrm{L}^{-1}$ alpha-pinene, $157 \mathrm{mg} \mathrm{L}^{-1}$ beta-pinene, $118 \mathrm{mg} \mathrm{L}^{-}$ 1 myrcene, $132 \mathrm{mg} \mathrm{L}^{-1}$ p-cymene, $159 \mathrm{mg} \mathrm{L}^{-1}$ citronellal, $182 \mathrm{mg} \mathrm{L}^{-1}$ L-menthol, $185 \mathrm{mg} \mathrm{L}^{-1}$ borneol and $128 \mathrm{mg} \mathrm{L}^{-1}$ thymol in methanol.

For SPE $500 \mu \mathrm{L}$ of the mixed standard were diluted with $9.5 \mathrm{~mL}$ water to reduce the methanol concentration to $5 \%$. The resulting $10 \mathrm{~mL}$ standard solution was used for one extraction.

Furthermore a mouthwash, two bath additives and cough drops were purchased in the local supermarket.

Mouthwash: $15 \mathrm{~mL}$ of the mouthwash were filled up to $250.0 \mathrm{~mL}$ with ultrapure water instead of $5 \%$ methanolic solution, as the mouthwash contains about $20 \%$ ethanol. For each extraction procedure $10.0 \mathrm{~mL}$ of this solution were used; thymol and menthol were quantified.

Bath additive 1: $5 \mathrm{~g}$ of the bath additive were filled up to $250 \mathrm{~mL}$ with $5 \%$ methanolic solution and was allowed to stand overnight to achieve phase separation. For each extraction procedure $10.0 \mathrm{~mL}$ of the aqueous phase were used; thymol and p-cymene were quantified.

Bath additive 2: $5 \mathrm{~g}$ of the bath additive were filled up to $250 \mathrm{~mL}$ with $5 \%$ methanolic solution. For each extraction procedure $10.0 \mathrm{~mL}$ of this solution were used; menthol and p-cymene were quantified.

Cough drops: 5 cough drops (3.6 g each) were dissolved in $200 \mathrm{~mL}$ of ultrapure water. $25 \mathrm{~mL}$ methanol was added and the flask was filled up to $500.0 \mathrm{~mL}$ with ultrapure water. For each extraction procedure $10.0 \mathrm{~mL}$ of this solution were used; menthol was quantified.

\subsection{Solid-Phase Extraction (SPE)}

Automated SPE: Solid-phase extraction was done employing the MEA Personal Purification System ${ }^{\circledR}$ (PhyNexus $^{\mathrm{TM}}$ Inc., San Jose, CA, USA) [14] using tips containing $160 \mu \mathrm{L}$ SPE-material (Fig. 1). $10 \mathrm{~mL}$ prepared sample solution were pipetted into a $2.2 \mathrm{~mL}$ storage plate (Thermo Fisher Scientific, Dreieich, Germany) as depicted in Fig. (2) (1 mL per well). SPE tips were manually installed on the pipette head of the Personal Purification System $\left.{ }^{(}\right)$ two materials, six tips of each material - and the extraction method was started. Exact method executed with the Personal Purification System ${ }^{\circledR}$ is provided in Table 1. The loading step took up $1 \mathrm{~mL}$ with a speed of $0.5 \mathrm{~mL} \mathrm{~min}^{-1}$ and a delay of $20 \mathrm{sec}$. Expelling step blew out $1 \mathrm{~mL}$ with a speed of $0.4 \mathrm{~mL} \mathrm{~min}^{-1}$ and a delay of $180 \mathrm{sec}$. The drying step took up and blew out $1 \mathrm{~mL}$ with a speed of $30 \mathrm{~mL} \mathrm{~min}^{-1}$ and a delay of $1 \mathrm{sec}$.

Finally the analytes were manually eluted from the tips using $700 \mu \mathrm{L}$ methanol into a $1 \mathrm{~mL}$ volumetric flask. The volumetric flask was filled with methanol to the calibrating mark and the resulting sample analyzed by GC-MS. Total extraction procedure of the Personal Purification System ${ }^{\circledR}$ took 5 h 15 min. Extraction was done 6 times with each SPE material. Tips were purchased from PhyNexus ${ }^{\mathrm{TM}}$ Inc. (San Jose, CA, USA) except for Strata-X ${ }^{\circledR}$, Cellulose, Florisil and Davisil which were manually packed into empty tips from PhyNexus ${ }^{\text {TM }}$ Inc. Strata- $X^{\circledR}$ was gained out of SPE cartridges (Phenomenex, Aschaffenburg, Germany). Cellulose (Avicel $^{\circledR}$, PH 101, Fluka, Steinheim, Germany), Florisil 

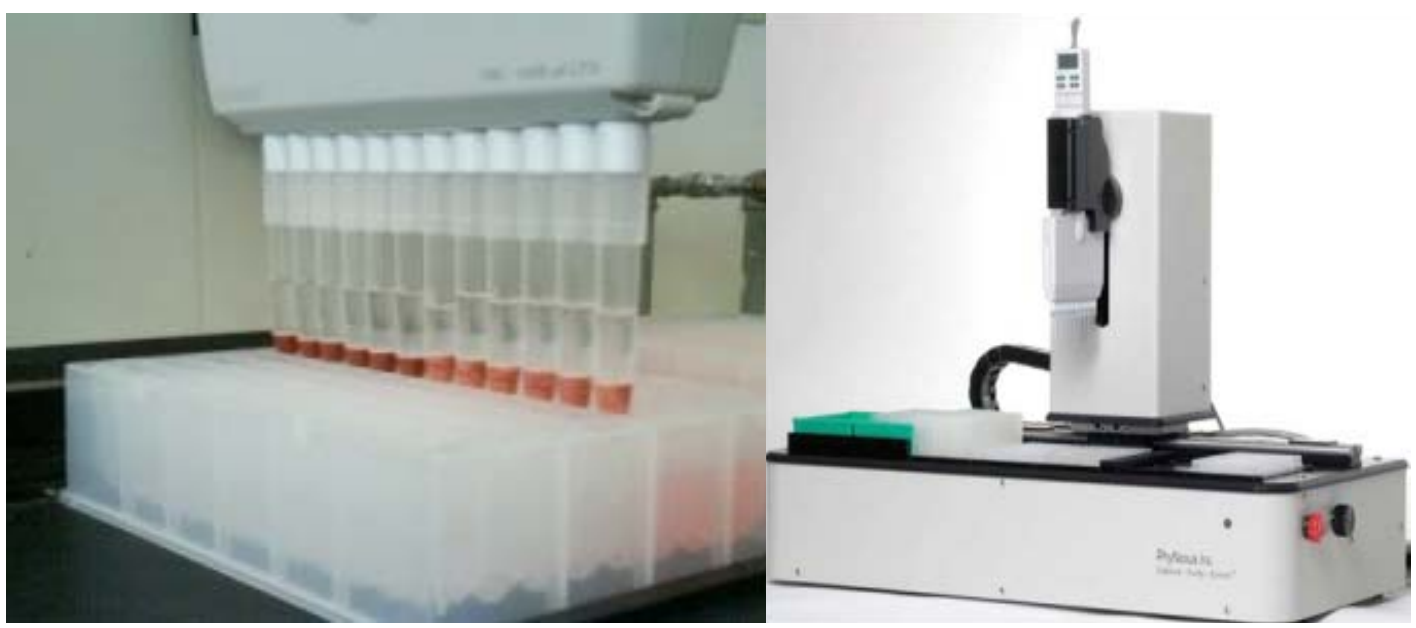

Fig. (1). MEA Personal Purification System ${ }^{\circledR}$ (PhyNexus Inc., San Jose, CA, USA).

(Carl Roth $\mathrm{GmbH}+$ Co.KG, Karlsruhe, Germany), Davisil (Grace Davison, Worms, Germany) were purchased as bulk material.

\subsection{Variations of Solid-Phase Extraction Procedure}

To study the effects of extraction parameters, method and protocol were changed in different points: Each well extracted once and twice, uptake of only $0.8 \mathrm{~mL}$ sample, skipping of drying step and changes in loading and expelling speed.

Each well extracted once: Extraction procedure was mainly the same as described before (Table 1) with the difference that each well was only extracted once. This way the extraction time was reduced to $2 \mathrm{~h} 07 \mathrm{~min}$. Extraction was done with $\mathrm{C}_{18}$ Hydra and $\mathrm{C}_{18} \mathrm{ec}$.

Each well extracted twice: Extraction procedure was mainly the same as with normal extraction (Table 1) with the

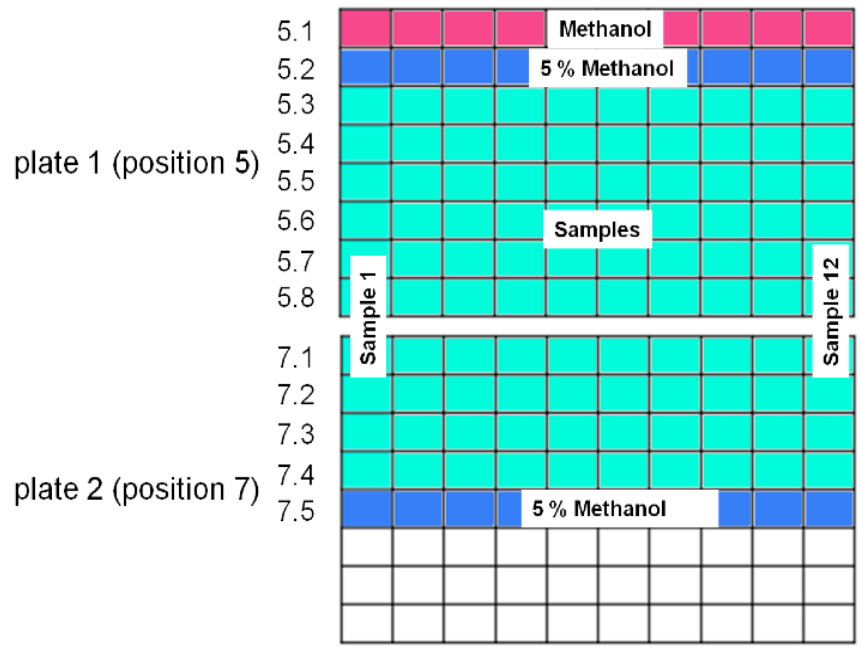

Fig. (2). Schematic view of storage plate.

Table 1. Method Used for Automated SPE

\begin{tabular}{|c|c|c|c|}
\hline Row & Filling & Repetitions & Step \\
\hline \hline 5.1 & $1 \mathrm{~mL}$ methanol & 2 & conditioning \\
\hline 5.2 & $1 \mathrm{~mL}$ water with $5 \%$ methanol & 3 & conditioning \\
\hline 5.3 & $1 \mathrm{~mL}$ sample & 3 & extraction (loading + expelling) \\
\hline 5.4 & $1 \mathrm{~mL}$ sample & 3 & extraction \\
\hline 5.5 & $1 \mathrm{~mL}$ sample & 3 & extraction \\
\hline 5.6 & $1 \mathrm{~mL}$ sample & 3 & extraction \\
\hline 5.7 & $1 \mathrm{~mL}$ sample & 3 & extraction \\
\hline 5.8 & $1 \mathrm{~mL}$ sample & 3 & extraction \\
\hline 7.1 & $1 \mathrm{~mL}$ sample & 3 & extraction \\
\hline 7.2 & $1 \mathrm{~mL}$ sample & 3 & extraction \\
\hline 7.3 & $1 \mathrm{~mL}$ sample & 3 & washing \\
\hline 7.4 & $1 \mathrm{~mL}$ sample & 30 & drying \\
\hline 7.5 & $1 \mathrm{~mL}$ water with $5 \%$ methanol & air & \\
\hline
\end{tabular}


difference that each well was only extracted twice. This led to a reduced extraction time of $3 \mathrm{~h} 41 \mathrm{~min}$. Extraction was done with $\mathrm{C}_{18}$ Hydra and $\mathrm{C}_{18}$ ec.

Uptake of $0.8 \mathrm{~mL}$ sample: Extraction procedure was mainly the same as with normal extraction with the difference that only $0.8 \mathrm{~mL}$ sample was taken up. This resulted in a reduced extraction time of $4 \mathrm{~h} 39 \mathrm{~min}$. The extraction was done with $\mathrm{C}_{18}$ Hydra and $\mathrm{C}_{18} \mathrm{ec}$.

Skipping of drying step: Extraction procedure was mainly the same as with normal extraction with the difference that the drying step was skipped. The extraction was done with $\mathrm{C}_{18}$ Hydra and $\mathrm{C}_{18}$ ec and took $5 \mathrm{~h} 13 \mathrm{~min}$.

Changes in loading and expelling speed: Extraction procedure was mainly the same as with normal extraction with the difference that the loading speed was changed to $0.5 \mathrm{~mL} \mathrm{~min}^{-1}$ and the expelling speed to $0.4 \mathrm{~mL} \mathrm{~min}^{-1}$. This led to a reduced extraction time of $2 \mathrm{~h} 38 \mathrm{~min}$. The extraction was done with $\mathrm{C}_{18}$ Hydra and $\mathrm{C}_{18} \mathrm{ec}$.

\subsection{Manual Solid-Phase Extraction}

To compare recovery rates all extractions were done manually, too, employing classic cartridges and tips. In principal extraction was done the same way as with the

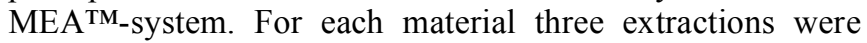
performed. Cartridges including the stationary phase were purchased from Macherey-Nagel except Strata- $X^{\circledR}$ which was purchased from Phenomenex. Manual extraction with cartridges was done with a syringe and adapter. For the manual extraction of tips, liquid was pipetted on the column bed of the tip and pushed through with a fitting Rainin ${ }^{\circledR}$ pipette (Mettler-Toledo, Gießen, Germany). SPE cartridges or tips were conditioned with $1 \mathrm{~mL}$ of methanol and $1 \mathrm{~mL}$ water containing $5 \%$ methanol. After that $1 \mathrm{~mL}$ of diluted standard solution was pipetted on top of the column bed and pushed through. The same was done with the remaining $9 \mathrm{~mL}$. In the next step the cartridges or tips were washed with $1 \mathrm{~mL}$ of water containing $5 \%$ methanol. Finally the analytes were eluted from the cartridges or tips using $700 \mu \mathrm{L}$ methanol into a $1 \mathrm{~mL}$ volumetric flask. The volumetric flask was filled with methanol to the calibrating mark and the resulting sample analyzed by gas chromatography mass spectrometry (GC-MS).

\subsection{Gas Chromatography - Mass Spectrometry (GC- MS) Measurements}

The GC-MS method used for recovery experiments was developed by the authors. Measurements were carried out on an Agilent $6890 \mathrm{~N}$ gas chromatograph coupled to a 5973 inert mass selective detector (Agilent Technologies, Waldbronn, Germany) equipped with a Combi PAL autosampler (CTC, Zwingen, Switzerland). For data acquisition MSD ChemStation E.02.01.1177 was used (Agilent Technologies, Waldbronn, Germany). Separation was done on a HP-20M (Carbowax 20M) column; $25 \mathrm{~m} \mathrm{x} 0.20 \mathrm{~mm} \times 0.2 \mu \mathrm{m}$ (Hewlett-Packard, Palo Alto, CA, USA). The temperature program used was $45^{\circ} \mathrm{C}-0.5 \mathrm{~min}-10^{\circ} \mathrm{C} \mathrm{m^{-1 }}-180^{\circ} \mathrm{C}-$ $20^{\circ} \mathrm{C} / \mathrm{min}^{-1}-200^{\circ} \mathrm{C}-3.0 \mathrm{~min}$; the carrier gas flow (helium) was $0.5 \mathrm{~mL} \mathrm{~min}^{-1}$. Injected sample volume: $1 \mu \mathrm{L}$; split ratio: $10: 1$; inlet temperature $280^{\circ} \mathrm{C}$. The sample was analyzed in the full scan mode using a mass range of 100-250 Th after electron impact ionization $(70 \mathrm{eV})$. The interface and ion source temperatures were $250{ }^{\circ} \mathrm{C}$ and $230{ }^{\circ} \mathrm{C}$, respectively, the quad temperature $150{ }^{\circ} \mathrm{C} .100 \mu \mathrm{L}$ of sample were mixed with $10 \mu \mathrm{L}$ of an internal standard solution containing $25.05 \mathrm{mg} \mathrm{mL}^{-1}$ o-cresol. For the quantification of thymol the ion with $135 \mathrm{Th}$, for borneol $95 \mathrm{Th}$, for menthol $71 \mathrm{Th}$, for citronellal $69 \mathrm{Th}$, for p-cymene $119 \mathrm{Th}$, for myrcene $93 \mathrm{Th}$ and for the external standard o-cresol $108 \mathrm{Th}$ were used.

The method was validated regarding selectivity, linearity, limit of quantification and precision. Method linearity was given between $2.8 \mathrm{mg} \mathrm{L}^{-1}$ and $136 \mathrm{mg} \mathrm{L}^{-1}$ for thymol, between $8 \mathrm{mg} \mathrm{L}^{-1}$ and $200 \mathrm{mg} \mathrm{L}^{-1}$ for borneol, between $3.8 \mathrm{mg} \mathrm{L}^{-1}$ and $180 \mathrm{mg} \mathrm{L}^{-1}$ for menthol, between $3.3 \mathrm{mg} \mathrm{L}^{-1}$ and $157 \mathrm{mg} \mathrm{L}^{-1}$ for citronellal, between $2.9 \mathrm{mg} \mathrm{L}^{-1}$ and $138 \mathrm{mg} \mathrm{L}^{-1}$ for p-cymene and between $2.6 \mathrm{mg} \mathrm{L}^{-1}$ and $127 \mathrm{mg} \mathrm{L}^{-1}$ for myrcene with $\mathrm{R}^{2} \geq 0.99$. Limit of quantification was set to $2.8 \mathrm{mg} \mathrm{L}^{-1}$ for thymol, $8 \mathrm{mg} \mathrm{L}^{-1}$ for borneol, $3.8 \mathrm{mg} \mathrm{L}^{-1}$ for menthol, $3.3 \mathrm{mg} \mathrm{L}^{-1}$ for citronellal, $2.9 \mathrm{mg} \mathrm{L}^{-1}$ for p-cymene and $2.6 \mathrm{mg} \mathrm{L}^{-1}$ for myrcene. From a sixfold injection a coefficient of variation of $3.2 \%$ resulted for myrcene, $4.7 \%$ for p-cymene, $3.6 \%$ for citronellal, $3.5 \%$ for menthol, $3.4 \%$ for borneol and $4.4 \%$ for thymol. Accuracy data is not given, as the complete work consists of recovery experiments.

\section{RESULTS AND DISCUSSION}

In the first step the system was adopted, evaluated and validated applying standards and mixtures thereof. In the following experiments the optimized protocol was extrapolated to the analysis of real samples.

\subsection{Enrichment and Analysis of Standards}

The gas chromatogram of mouthwash after extraction with Strata- $X^{\circledR}$ is depicted in Fig. (3). In principal separation is achieved in order of the analytes' polarity: myrcene, $p$ cymene, citronellal, menthol, borneol, o-cresol (internal standard) and thymol. Recovery rates for high concentration are given in Table 2, while those for low concentration can be deduced from Table 3 .

At high concentration thymol, borneol, menthol and citronellal showed highest recovery rates for Strata- $X^{\circledR}$. For thymol second best material was $\mathrm{C}_{18}$ ec closely followed by $\mathrm{C}_{8}$, for borneol $\mathrm{C}_{18}$ Hydra followed by $\mathrm{C}_{4}$. For menthol second best material was $\mathrm{C}_{18}$ ec closely followed by $\mathrm{C}_{8}$ and $\mathrm{C}_{18}$ Hydra, for citronellal $\mathrm{C}_{8}$ and $\mathrm{C}_{18}$ ec closely followed by $\mathrm{C}_{18}$ Hydra. For p-cymene only $\mathrm{C}_{18} \mathrm{Hydra}$ and for myrcene $\mathrm{C}_{18}$ Hydra and Davisil showed recovery $\geq 10 \%$. alpha-Pinene and beta-pinene were not interpreted because of improper peak shape.

Low concentration resulted for thymol, borneol, menthol and citronellal in highest recovery rates with Strata- $X^{\circledR}$. For thymol second best material was $\mathrm{C}_{18}$ ec closely followed by $\mathrm{C}_{18}$ Hydra and $\mathrm{C}_{6} \mathrm{H}_{11}$ ec, for borneol by $\mathrm{C}_{18}$ Hydra and $\mathrm{C}_{18}$ ec. For menthol second best material was $\mathrm{C}_{18}$ Hydra closely followed by $\mathrm{C}_{18}$ ec and $\mathrm{C}_{6} \mathrm{H}_{11}$ ec. Citronellal showed second best result with $\mathrm{C}_{8}$ and $\mathrm{C}_{18}$ ec closely followed by $\mathrm{C}_{18}$ Hydra and $\mathrm{C}_{6} \mathrm{H}_{11}$ ec. For p-cymene and myrcene achieved recovery rates were very low with all materials $(<10 \%)$. 
Abundance

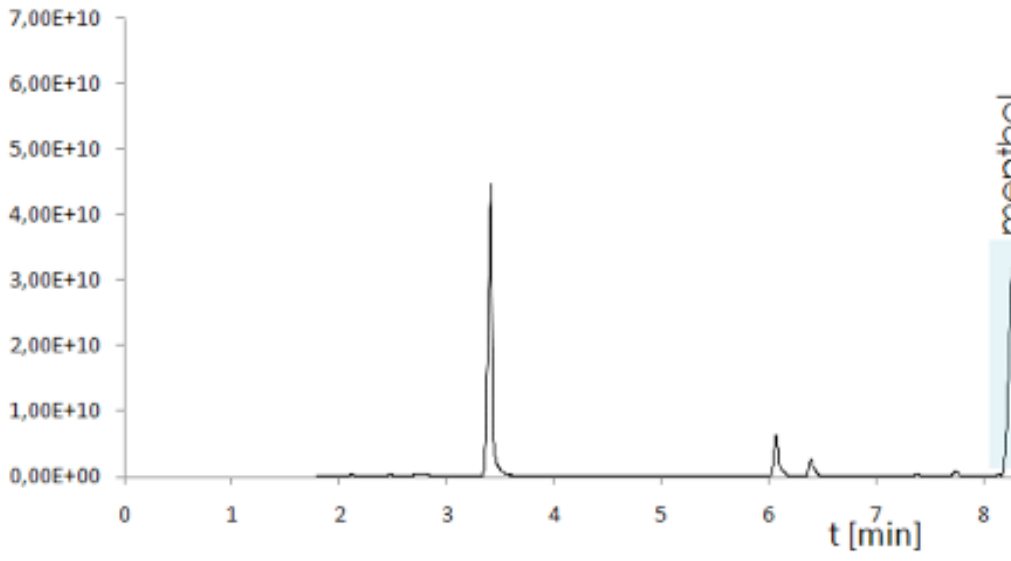

\section{mouthwash}

Fig. (3). Chromatogram of mouthwash extracted with Strata-X.

Table 2. Recovery Rates of High Concentrated Monoterpene Standards in \% Including Standard Deviation and Coefficient of Variation in Italics

\begin{tabular}{|c|c|c|c|c|c|c|}
\hline & Myrcene & p-Cymene & Citronellal & Menthol & Borneol & Thymol \\
\hline $\mathrm{C}_{2}$ & $<10$ & $<10$ & $<10$ & $<10$ & $20 \pm 4(19)$ & $<10$ \\
\hline $\mathrm{C}_{8}$ & $<10$ & $<10$ & $34 \pm 2(7)$ & $64 \pm 9(14)$ & $50 \pm 8(16)$ & $62 \pm 8(13)$ \\
\hline $\mathrm{C}_{18}$ Hydra & $12 \pm 1(7)$ & $12 \pm 1(6)$ & $24 \pm 2(7)$ & $62 \pm 6(10)$ & $58 \pm 10(17)$ & $49 \pm 8(17)$ \\
\hline $\mathrm{C}_{6} \mathrm{H}_{11} \mathrm{ec}$ & $<10$ & $<10$ & $25 \pm 3(12)$ & $48 \pm 5(10)$ & $15 \pm 3(22)$ & $45 \pm 4(9)$ \\
\hline $\mathrm{C}_{6} \mathrm{H}_{5}$ & $<10$ & $<10$ & $15 \pm 5(31)$ & $21 \pm 5(25)$ & $<10$ & $16 \pm 4(25)$ \\
\hline $\mathrm{CN}$ & $<10$ & $<10$ & $<10$ & $<10$ & $27 \pm 1(4)$ & $<10$ \\
\hline $\mathrm{NH}_{2} / \mathrm{C}_{18}$ & $<10$ & $<10$ & $19 \pm 2(10)$ & $39 \pm 3(7)$ & $<10$ & $27 \pm 2(6)$ \\
\hline HR-P & $<10$ & $<10$ & $19 \pm 3(14)$ & $37 \pm 5(14)$ & $<10$ & $39 \pm 6(15)$ \\
\hline PA & $<10$ & $<10$ & $<10$ & $<10$ & $<10$ & $16 \pm 1(6)$ \\
\hline Strata-X ${ }^{\circledR}$ & $<10$ & $<10$ & $58 \pm 5(9)$ & $88 \pm 6(7)$ & $87 \pm 6(7)$ & $92 \pm 7(7)$ \\
\hline Cellulose & $<10$ & $<10$ & $<10$ & $<10$ & $<10$ & $<10$ \\
\hline Davisil & $15 \pm 2(13)$ & $<10$ & $<10$ & $<10$ & $<10$ & $<10$ \\
\hline Florisil & $<10$ & $<10$ & $<10$ & $<10$ & $20 \pm 4(19)$ & $<10$ \\
\hline
\end{tabular}

From those results one can see that in general especially polymeric materials like Strata- $X^{\circledR}$, Easy, HR-P and PA have low standard deviations and coefficients of variation in comparison to silica based materials. Furthermore results show that taking standard deviation into account, recovery rates were in the same range for low and high concentration. Thus breakthrough of analytes resulting from exceeding capacity of column bed can be excluded. Only in case of borneol extraction with Strata- $\mathrm{X}^{\circledR}$ standard deviation cannot compensate the difference in recovery rates, but most probably is a statistic variation.
The results clearly show that $\mathrm{C}_{18}$ is not the most effective SPE material for this task. Furthermore it can be seen that recovery rates for thymol, borneol and menthol are much higher than for myrcene, p-cymene and citronellal. The reason may be the higher volatility of the more nonpolar compounds. As the extraction took quite a long time (5 h $15 \mathrm{~min}$ ) analytes had the opportunity to vaporize. Another explanation for lower recovery of more nonpolar compounds is the low solubility of the analytes in the sample solution, which consisted of water with 5\% methanol. Analytes can have precipitated before or during SPE 
Table 3. Recovery Rates of Low Concentrated Monoterpene Standards in \% Including Standard Deviation and Coefficient of Variation in Italics (Myrcene and p-Cymene in All Cases < 10\%)

\begin{tabular}{|c|c|c|c|c|}
\hline & Citronellal & Menthol & Borneol & Thymol \\
\hline $\mathrm{C}_{2}$ & $<10$ & $<10$ & $<10$ & $<10$ \\
\hline $\mathrm{C}_{8}$ & $32 \pm 5(16)$ & $53 \pm 10(19)$ & $48 \pm 10(22)$ & $53 \pm 11(21)$ \\
\hline $\mathrm{C}_{18}$ Hydra & $27 \pm 5(19)$ & $69 \pm 9(13)$ & $58 \pm 11(19)$ & $58 \pm 11(19)$ \\
\hline $\mathrm{C}_{6} \mathrm{H}_{11} \mathrm{ec}$ & $26 \pm 5(18)$ & $62 \pm 12(20)$ & $48 \pm 8(16)$ & $56 \pm 11(19)$ \\
\hline $\mathrm{C}_{6} \mathrm{H}_{5}$ & $13 \pm 3(24)$ & $20 \pm 2(11)$ & $17 \pm 2(9)$ & $17 \pm 1(8)$ \\
\hline $\mathrm{CN}$ & $<10$ & $<10$ & $<10$ & $<10$ \\
\hline $\mathrm{NH}_{2} / \mathrm{C}_{18}$ & $23 \pm 6(27)$ & $47 \pm 14(30)$ & $38 \pm 16(43)$ & $39 \pm 16(42)$ \\
\hline HR-P & $19 \pm 2(9)$ & $36 \pm 3(8)$ & $35 \pm 3(7)$ & $39 \pm 3(7)$ \\
\hline PA & $<10$ & $<10$ & $<10$ & $16 \pm 2(10)$ \\
\hline Strata- $X^{\circledR}$ & $50 \pm 3(5)$ & $76 \pm 4(5)$ & $94.8 \pm 4(4)$ & $94 \pm 3(3)$ \\
\hline Cellulose & $<10$ & $<10$ & $<10$ & $<10$ \\
\hline Davisil & $<10$ & $<10$ & $<10$ & $<10$ \\
\hline Florisil & $<10$ & $<10$ & $<10$ & $<10$ \\
\hline
\end{tabular}

cleanup. Strata- $X^{\circledR}$ shows highest recovery of all tested sorbents for each standard analyte. This might be based on the special chemistry combined the high specific surface [15]. Wissiack et al. obtained similar results, when they compared different sorbents for online SPE coupled with liquid chromatography - mass spectrometry of phenols. They obtained high recoveries with good relative standard deviations with Oasis ${ }^{\circledR}$ HLB compared to silica based sorbents or conventional polymer based sorbents [16].

Strata- $\mathrm{X}^{\circledR}$ offers $\pi-\pi$ bonding due to the phenyl ring in the ligand. This can increase recovery of aromatic analytes, e.g. p-cymene and thymol. Hydrophobic interactions of the styrene-divinylbenzene backbone leads to high recovery of neutral hydrophobic analytes, e.g. monoterpenes. The pyrrolidone ligand is able to form hydrogen bonds with analytes containing hydroxyl groups, e.g. thymol, borneol or menthol. Furthermore it can interact with acidic compounds. Additionally the pyrrolidone ligand can participate in dipoledipole interactions, e.g. with aldehydes like citronellal. Highest recovery rates were observed with thymol and borneol. Thymol is able to use four interactions: $\pi-\pi$ bonding, hydrophobic interactions, hydrogen bonds and interaction with acidic compounds. As thymol is a derivate of phenol having the character of a weak acid it can interact with the basic pyrrolidone ligand. Borneol can interact by hydrogen bonding and hydrophobic interaction.

$\mathrm{C}_{2}$ and $\mathrm{C}_{4}$ are traditionally used for the extraction of analytes, which are too strongly retained with $\mathrm{C}_{18}$-silica and show, as expected, low recovery rates for all analytes. $\mathrm{C}_{8}$ is used for the same analytes as $\mathrm{C}_{18}$, but as the alkyl chain is shorter, it offers the possibility of polar interactions with the free silanols on the silica surface. But in this experiment, this does not have a great influence as the recovery rates for $\mathrm{C}_{8}$, $\mathrm{C}_{18}$ ec and $\mathrm{C}_{18}$ Hydra do not differ, especially when taking standard deviation into account. $\mathrm{C}_{6} \mathrm{H}_{11}$ ec showed recovery rates in the same range as $\mathrm{C}_{8}, \mathrm{C}_{18}$ ec and $\mathrm{C}_{18}$ Hydra, even for thymol, although it is recommended for the extraction of phenols from water. Surprisingly $\mathrm{C}_{6} \mathrm{H}_{5}$ showed lower recovery than $\mathrm{C}_{8}, \mathrm{C}_{18}$ ec and $\mathrm{C}_{18}$ Hydra, so $\pi$ - $\pi$ bonding seems to have lower influence on recovery than hydrophobic interaction. $\mathrm{CN}, \mathrm{OH}, \mathrm{NO}_{2}, \mathrm{PA}$, Cellulose, Davisil and Florisil are normal phase sorbents, which were used in reversed-phase (RP) mode and thus it is no surprise that they show quite low recovery. According to Machery-Nagel ${ }^{\mathbb{R}}$, $s$ product information, HR-P is a poly(styrene-codivinylbenzene) polymer with a high specific surface of $1200 \mathrm{~m}^{2} / \mathrm{g}$ recommended for the extraction of aromatic compounds and phenols from water. Easy is a polystyrenedivinylbenzene polymer $\left(650-700 \mathrm{~m}^{2} / \mathrm{g}\right)$ functionalized with a weak ion exchanger to be more hydrophilic and is recommended for the extraction of polar phenols from water. In comparison to HR-P, Easy did not show better recovery due to its ion exchange capacity.

\subsection{Variation of Extraction Procedure}

Tables $\mathbf{4}$ and $\mathbf{5}$ show the recovery rates obtained with $\mathrm{C}_{18}$ Hydra and $\mathrm{C}_{18}$ ec using the different SPE methods as described in the experimental section 2.4.

For $\mathrm{C}_{18}$ Hydra in case of thymol, borneol and menthol extracting each well only one time and extraction of $0.8 \mathrm{~mL}$ led to lower recovery rates in comparison to the "normal" 
Table 4. Recovery Rates of Monoterpenes Achieved for Different Extraction Variations with $\mathrm{C}_{18} \mathrm{Hydra}$ in $\%$ Including Standard Deviation and Coefficient of Variation in Italics (Myrcene and p-Cymene in all Cases < 10\%)

\begin{tabular}{|c|c|c|c|c|}
\hline & Citronellal & Menthol & Borneol & Thymol \\
\hline \hline normal & $27 \pm 5(19)$ & $69 \pm 9(13)$ & $58 \pm 11(19)$ & $58 \pm 11(19)$ \\
\hline 2 x extraction & $31 \pm 4(13)$ & $76 \pm 10(13)$ & $64 \pm 13(21)$ & $65 \pm 15(23)$ \\
\hline $1 \times$ extraction & $44 \pm 3(6)$ & $54 \pm 6(11)$ & $39 \pm 8(19)$ & $40 \pm 8(19)$ \\
\hline 0.8 mL extraction & $32 \pm 6(19)$ & $49 \pm 10(21)$ & $40 \pm 12(31)$ & $41 \pm 13(32)$ \\
\hline fast extraction & $31 \pm 2(6)$ & $77 \pm 2(2)$ & $63 \pm 2(4)$ & $65 \pm 3(4)$ \\
\hline without drying & $16 \pm 1(5)$ & $65 \pm 2(3)$ & $52 \pm 4(7)$ & $53 \pm 5(9)$ \\
\hline
\end{tabular}

Table 5. Recovery Rates of Monoterpenes Achieved with Different Extraction Variations for $\mathrm{C}_{18}$ ec in \% Including Standard Deviation and Coefficient of Variation in Italics (Myrcene in All Cases $<10 \%$ )

\begin{tabular}{|c|c|c|c|c|c|}
\hline & p-Cymene & Citronellal & Menthol & Borneol & Thymol \\
\hline \hline normal & $<10$ & $31 \pm 5(16)$ & $64 \pm 9(14)$ & $57 \pm 12(21)$ & $61 \pm 12(20)$ \\
\hline $2 \times$ extraction & $<10$ & $35 \pm 2(5)$ & $82 \pm 3(3)$ & $73 \pm 10(14)$ & $81 \pm 9(11)$ \\
\hline $1 \times$ extraction & $<10$ & $38 \pm 6(15)$ & $47 \pm 8(17)$ & $40 \pm 10(26)$ & $44 \pm 10(23)$ \\
\hline 0.8 mL extraction & $<10$ & $39 \pm 7(18)$ & $59 \pm 10(17)$ & $51 \pm 9(17)$ & $54 \pm 9(16)$ \\
\hline fast extraction & $11 \pm 1(10)$ & $37 \pm 1(3)$ & $88 \pm 2(2)$ & $83 \pm 4(4)$ & $90 \pm 3(3)$ \\
\hline without drying & $<10$ & $16 \pm 4(23)$ & $73 \pm 8(12)$ & $69 \pm 11(16)$ & $77 \pm 11(15)$ \\
\hline
\end{tabular}

procedure. Taking standard deviation into account, differences are compensated for the most part. Regarding standard deviation no significant difference was obtained in case of citronellal. Skipping of drying step and fast extraction resulted in most cases in lower coefficients of variation. Best results were achieved with fast extraction as it resulted in high recovery and low coefficient of variation. For $\mathrm{C}_{18} \mathrm{ec}$ in case of thymol, borneol and menthol fast extraction led to higher recovery rates in comparison to the "normal" procedure. The same was obtained for two times extraction, but standard deviations are compensating the differences. Extracting only once or extracting $0.8 \mathrm{~mL}$ resulted in reduced recovery rates. Regarding standard deviation no significant difference was obtained in case of citronellal. Fast extraction additionally led to lower coefficient of variation.

\subsection{Manual Solid-Phase Extraction}

In the first step cartridges and in the following tips were employed for SPE.

\subsubsection{Cartridges}

Table 6 gives the results obtained by manual extraction with classical SPE cartridges. High recovery rates with nearly equal results were obtained with $\mathrm{C}_{4}, \mathrm{C}_{8}, \mathrm{C}_{18}$, $\mathrm{C}_{18}$ Hydra, $\mathrm{C}_{18}$ ec, $\mathrm{C}_{6} \mathrm{H}_{11}$ ec and $\mathrm{C}_{6} \mathrm{H}_{5}$ and Strata- $\mathrm{X}^{\circledR}$. HR-X did not deliver as high results as Strata- $X^{\circledR}$ with only $76 \%$ recovery for thymol, $81 \%$ for menthol and $77 \%$ for borneol. Even in the manual operation mode, overall recovery rates for myrcene and p-cymene were very low. The difference in standard deviation and coefficient of variation obtained with automation was not found in the same dimensions, especially in case of menthol, borneol and thymol.
Manual extraction with cartridges led to high recoveries with a wide range of sorbents $\left(\mathrm{C}_{4}, \mathrm{C}_{8}, \mathrm{C}_{18}, \mathrm{C}_{18}\right.$ Hydra, $\mathrm{C}_{18} \mathrm{ec}$, $\mathrm{C}_{6} \mathrm{H}_{11} \mathrm{ec}, \mathrm{C}_{6} \mathrm{H}_{5}$ and Strata- $\left.\mathrm{X}^{\mathbb{R}}\right)$, especially for thymol, borneol and menthol. So here the hydrophobic interactions of $\mathrm{C}_{4}, \mathrm{C}_{8}, \mathrm{C}_{18}, \mathrm{C}_{18}$ Hydra, $\mathrm{C}_{18}$ ec, $\mathrm{C}_{6} \mathrm{H}_{11}$ ec work as well as the additional $\pi$ - $\pi$ interactions of $\mathrm{C}_{6} \mathrm{H}_{5}$ and the many possible interactions of Strata- $\mathrm{X}^{\circledR}$ already discussed in the previous chapters. 3 Endcapping does not seem to have significant influence on recovery of all of the analytes as recovery rates achieved with $\mathrm{C}_{18} \mathrm{Hydra}, \mathrm{C}_{18}$ and $\mathrm{C}_{18}$ ec show similar resuts. As expected, the extraction with the normalphase(NP) sorbents $\mathrm{OH}, \mathrm{NO}_{2}$, Florisil, $\mathrm{SiOH}$ and $\mathrm{NH}_{2}$ did not reach high recoveries. $\mathrm{CN}$ is a normal-phase sorbent that can also be used in reversed-phase mode. Due to its high electron density it can show selective interaction, but is less hydrophobic than $\mathrm{C}_{18}$ or $\mathrm{C}_{8}$ and is recommended for the extraction of lipophilic compounds from aqueous solutions, which are too strongly retained with $\mathrm{C}_{8}$ or $\mathrm{C}_{18}$. As the extraction of the applied analytes works well with $\mathrm{C}_{18}$ and $\mathrm{C}_{8}$, the hydrophobic interaction of $\mathrm{CN}$ seems to be too low to reach satisfying recovery. HR-X, a hydrophobic poly(styrene-co-divinylbenzene) polymer, which is recommended for the extraction of phenols from water, achieved moderate results in case of thymol, borneol and menthol. But as the exact composition and modification of this material is not available, further discussion would be wild guess. HR-P, a poly(styrene-co-divinylbenzene) copolymer, did only reach moderate recovery.

\subsubsection{Tips}

Table 7 gives the results obtained by manual extraction with SPE tips. For thymol, borneol and menthol very high recovery rates of about $100 \%$ were found with $\mathrm{C}_{4}, \mathrm{C}_{8}$, 
Table 6. Recovery Rates of Monoterpene Standards in \% Including Standard Deviation and Coefficient of Variation in Italics (Myrcene and p-Cymene in All Cases < 10\%) for Manual Extraction with Cartridges

\begin{tabular}{|c|c|c|c|c|c|c|}
\hline & Myrcene & p-Cymene & Citronellal & Menthol & Borneol & Thymol \\
\hline $\mathrm{C}_{2}$ & $<10$ & $<10$ & $<10$ & $<10$ & $<10$ & $<10$ \\
\hline $\mathrm{C}_{4}$ & $45 \pm 9(19)$ & $35 \pm 6(16)$ & $92 \pm 4(4)$ & $102 \pm 4(4)$ & $102 \pm 4(4)$ & $99 \pm 2(2)$ \\
\hline $\mathrm{C}_{8}$ & $39 \pm 3(8)$ & $48 \pm 5(11)$ & $93 \pm 6(6)$ & $105 \pm 3(3)$ & $105 \pm 3(3)$ & $103 \pm 3(3)$ \\
\hline $\mathrm{C}_{18}$ & $20 \pm 14(66)$ & $26 \pm 10(39)$ & $79 \pm 8(11)$ & $99 \pm 5(5)$ & $98 \pm 5(5)$ & $99 \pm 5(5)$ \\
\hline $\mathrm{C}_{18}$ Hydra & $32 \pm 2(5)$ & $42 \pm 2(5)$ & $90 \pm 4(5)$ & $103 \pm 3(3)$ & $103 \pm 3(3)$ & $104 \pm 4(4)$ \\
\hline $\mathrm{C}_{18} \mathrm{ec}$ & $31 \pm 1(2)$ & $38 \pm 1(4)$ & $89 \pm 4(4)$ & $104 \pm 5(5)$ & $101 \pm 4(4)$ & $100 \pm 5(4)$ \\
\hline $\mathrm{C}_{6} \mathrm{H}_{11} \mathrm{ec}$ & $23 \pm 2(7)$ & $37 \pm 2(5)$ & $83 \pm 1(2)$ & $97 \pm 2(2)$ & $98 \pm 2(2)$ & $94 \pm 2(2)$ \\
\hline $\mathrm{C}_{6} \mathrm{H}_{5}$ & $20 \pm 2(9)$ & $35 \pm 4(10)$ & $82 \pm 6(7)$ & $104 \pm 6(6)$ & $94 \pm 6(6)$ & $99 \pm 7(7)$ \\
\hline $\mathrm{CN}$ & $17 \pm 2(12)$ & $22 \pm 1(7)$ & $37 \pm 2(5)$ & $21 \pm 1(4)$ & $<10$ & $43 \pm 1(3)$ \\
\hline $\mathrm{OH}$ & $<10$ & $<10$ & $<10$ & $<10$ & $<10$ & $<10$ \\
\hline $\mathrm{NO}_{2}$ & $<10$ & $<10$ & $<10$ & $<10$ & $<10$ & $<10$ \\
\hline HR-P & $17 \pm 3(17)$ & $17 \pm 2(12)$ & $48 \pm 7(14)$ & $65 \pm 9(14)$ & $65 \pm 9(13)$ & $57 \pm 6(11)$ \\
\hline Strata- $X^{\circledR}$ & $30 \pm 4(13)$ & $44 \pm 1(3)$ & $82 \pm 1(2)$ & $93 \pm 2(2)$ & $93 \pm 2(2)$ & $94 \pm 2(2)$ \\
\hline Florisil & $<10$ & $<10$ & $21 \pm 1(3)$ & $13 \pm 1(6)$ & $<10$ & $<10$ \\
\hline HR-X & $<10$ & $<10$ & $32 \pm 3(10)$ & $77 \pm 5(7)$ & $81 \pm 5(6)$ & $76 \pm 5(6)$ \\
\hline $\mathrm{SiOH}$ & $<10$ & $<10$ & $<10$ & $<10$ & $<10$ & $<10$ \\
\hline $\mathrm{NH}_{2}$ & $<10$ & $<10$ & $<10$ & $<10$ & $<10$ & $<10$ \\
\hline
\end{tabular}

$\mathrm{C}_{18}$ Hydra, $\mathrm{C}_{18}$ ec, $\mathrm{C}_{6} \mathrm{H}_{11}$ ec, $\mathrm{C}_{6} \mathrm{H}_{5}, \mathrm{NH}_{2} / \mathrm{C}_{18}$ and Strata- $\mathrm{X}^{\circledR}$. Second best results around $70 \%$ gave Easy and HR-P. For citronellal recovery rates of about $100 \%$ (between 90 and $116 \%$ ) were achieved with $\mathrm{C}_{4}, \mathrm{C}_{8}, \mathrm{C}_{18}$ Hydra, $\mathrm{C}_{18} \mathrm{ec}$,
$\mathrm{C}_{6} \mathrm{H}_{11}$ ec and $\mathrm{C}_{6} \mathrm{H}_{5}$. Second best was Strata- $\mathrm{X}^{\circledR}$ with a recovery rate of about $80 \%$. Overall recovery rates for myrcene and p-cymene were very low also here in manual mode with SPE tips.

Table 7. Recovery Rates of Monoterpene Standards in \% Including Standard Deviation and Coefficient of Variation in Italics (Myrcene and p-Cymene in All Cases < 10\%) for Manual Extraction with Tips

\begin{tabular}{|c|c|c|c|c|c|c|}
\hline & Myrcene & p-Cymene & Citronellal & Menthol & Borneol & Thymol \\
\hline $\mathrm{C}_{2}$ & $<10$ & $<10$ & $<10$ & $<10$ & $<10$ & $<10$ \\
\hline $\mathrm{C}_{4}$ & $44 \pm 1(3)$ & $62 \pm 5(8)$ & $92 \pm 11(12)$ & $103 \pm 4(4)$ & $102 \pm 2(2)$ & $101 \pm 1(1)$ \\
\hline $\mathrm{C}_{8}$ & $68 \pm 8(11)$ & $68 \pm 12(18)$ & $107 \pm 18(17)$ & $108 \pm 15(14)$ & $109 \pm 15(14)$ & $110 \pm 14(13)$ \\
\hline $\mathrm{C}_{18}$ Hydra & $68 \pm 6(9)$ & $67 \pm 3(4)$ & $99 \pm 6(6)$ & $101 \pm 7(7)$ & $102 \pm 7(7)$ & $101 \pm 7(7)$ \\
\hline $\mathrm{C}_{6} \mathrm{H}_{5}$ & $53 \pm 0(0)$ & $23 \pm 6(25)$ & $92 \pm 10(11)$ & $107 \pm 5(5)$ & $95 \pm 4(4)$ & $107 \pm 3(3)$ \\
\hline $\mathrm{CN}$ & $12 \pm 1(7)$ & $12 \pm 1(6)$ & $14 \pm 2(13)$ & $<10$ & $<10$ & $13 \pm 2(17)$ \\
\hline $\mathrm{NH}_{2} / \mathrm{C}_{18}$ & $56 \pm 2(3)$ & $65 \pm 3(4)$ & $35 \pm 6(16)$ & $98 \pm 3(3)$ & $99 \pm 2(2)$ & $99 \pm 1(1)$ \\
\hline $\mathrm{OH}$ & $<10$ & $<10$ & $<10$ & $<10$ & $<10$ & $<10$ \\
\hline PA & $18 \pm 2(12)$ & $<10$ & $<10$ & $<10$ & $<10$ & $19 \pm 2(11)$ \\
\hline Strata- $^{\circledR}$ & $47 \pm 4(10)$ & $51 \pm 5(9)$ & $84 \pm 4(5)$ & $100 \pm 2(2)$ & $102 \pm 2(2)$ & $102 \pm 1(1)$ \\
\hline Cellulose & $<10$ & $<10$ & $<10$ & $<10$ & $<10$ & $<10$ \\
\hline Davisil & $<10$ & $<10$ & $<10$ & $<10$ & $<10$ & $<10$ \\
\hline Florisil & $<10$ & $<10$ & $14 \pm 2(15)$ & $<10$ & $<10$ & $<10$ \\
\hline
\end{tabular}


Comparable to manual extraction with cartridges for thymol, borneol and menthol a wide range of sorbents achieved recovery around $100 \%$. The mainly on hydrophobic interaction based $\mathrm{C}_{4}, \mathrm{C}_{8}, \mathrm{C}_{18}$ Hydra, $\mathrm{C}_{18} \mathrm{ec}, \mathrm{C}_{6} \mathrm{H}_{11}$ ec and HR-P as well as the mixed $\mathrm{NH}_{2} / \mathrm{C}_{18}$ sorbent and the "universal" Strata- $X^{\circledR}$. Easy and HR-P showed nearly equal recovery for all analytes, although HR-P is completely nonpolar, while Easy is modified to show hydrophilic character. For citronellal the hydrophobic interaction capacity of the silica based sorbents seems to work more efficiently than the many interactions of Strata- $X^{\circledR}$. Surprisingly recovery with $\mathrm{C}_{6} \mathrm{H}_{5}$ was quite low for $\mathrm{p}$ cymene, although $\pi-\pi$ interactions due to the aromatic ring systems should be expected.

\subsection{Real Sample Analysis}

In the following the above optimized protocols were applied for the analysis of real samples including mouthwash, bath additives and cough drops.

\subsubsection{Mouthwash}

Menthol and thymol were determined in mouthwash. Strata-X showed best results for thymol as well as menthol. Second best results were achieved with $\mathrm{C}_{8}$ followed by $\mathrm{C}_{18}$ ec, $\mathrm{C}_{6} \mathrm{H}_{11}$ ec and $\mathrm{C}_{18}$ Hydra (Table 8). In general, the SPE materials showed similar results as with standard solution.

Table 8. Thymol and Menthol Concentration in $\mathrm{mg} \mathrm{mL}^{-1}$ Found After Automated SPE of Mouthwash. Also Given are Standard Deviation and Coefficient of Variation in Italics

\begin{tabular}{|c|c|c|}
\hline & Menthol & Thymol \\
\hline $\mathrm{C}_{2}$ & $0.10 \pm 0.00(12.2)$ & $0.06 \pm 0.01(10.3)$ \\
\hline $\mathrm{C}_{4}$ & $0.29 \pm 0.04(39.3)$ & $0.18 \pm 0.06(34.9)$ \\
\hline $\mathrm{C}_{8}$ & $0.68 \pm 0.06(26.0)$ & $0.41 \pm 0.10(24.0)$ \\
\hline $\mathrm{C}_{18}$ Hydra & $0.54 \pm 0.05(21.9)$ & $0.33 \pm 0.05(16.7)$ \\
\hline $\mathrm{C}_{18} \mathrm{ec}$ & $0.64 \pm 0.05(22.4)$ & $0.38 \pm 0.05(13.8)$ \\
\hline $\mathrm{C}_{6} \mathrm{H}_{11} \mathrm{ec}$ & $0.60 \pm 0.04(21.6)$ & $0.36 \pm 0.05(14.7)$ \\
\hline $\mathrm{C}_{6} \mathrm{H}_{5}$ & $0.34 \pm 0.02(19.5)$ & $0.20 \pm 0.04(18.6)$ \\
\hline $\mathrm{CN}$ & $0.19 \pm 0.01(26.1)$ & $0.11 \pm 0.02(17.2)$ \\
\hline $\mathrm{NH}_{2} / \mathrm{C}_{18}$ & $0.40 \pm 0.04$ & $0.24 \pm 0.05(20.0)$ \\
\hline $\mathrm{OH}$ & $<$ LOQ & $0.01 \pm 0.00(20.9)$ \\
\hline $\mathrm{NO}_{2}$ & $0.10 \pm 0.00(29.3)$ & $0.06 \pm 0.02(28.2)$ \\
\hline Easy & $0.39 \pm 0.02(14.2)$ & $0.23 \pm 0.03(14.7)$ \\
\hline HR-P & $0.43 \pm 0.02(10.4)$ & $0.26 \pm 0.02(8.8)$ \\
\hline PA & $0.24 \pm 0.00(1.9)$ & $0.14 \pm 0.01(5.8)$ \\
\hline Strata-X & $0.97 \pm 0.01(4.0)$ & $0.58 \pm 0.02(3.1)$ \\
\hline Cellulose & $<$ LOQ & $<$ LOQ \\
\hline Davisil & $<$ LOQ & $0.01 \pm 0.01(58.2)$ \\
\hline Florisil & $0.05 \pm 0.01(20.2)$ & $0.03 \pm 0.00(16.6)$ \\
\hline
\end{tabular}

\subsubsection{Bath Additive}

In bath additive 1, thymol and p-cymene were determined (Table 9). For thymol Strata-X showed best results followed by $\mathrm{C}_{8}$ and $\mathrm{C}_{6} \mathrm{H}_{5}$. Similar results were achieved for p-cymene but the differences to other materials were quite small. In comparison to the experiment using pure standards, $\mathrm{CN}$ and $\mathrm{NO}_{2}$ had high recovery rates.

Table 9. Concentration of p-Cymene and Thymol in $\mathrm{mg} \mathrm{g}^{-1}$ Found After Automated SPE of Bath Additive 1. Also Given are Standard Deviation and Coefficient of Variation in Italics

\begin{tabular}{|c|c|c|}
\hline & p-Cymene & Thymol \\
\hline \hline $\mathrm{C}_{2}$ & $0.146 \pm 0.014(9.9)$ & $0.567 \pm 0.014(2.4)$ \\
\hline $\mathrm{C}_{4}$ & $0.139 \pm 0.035(24.9)$ & $0.645 \pm 0.138(21.5)$ \\
\hline $\mathrm{C}_{8}$ & $0.261 \pm 0.042(16.2)$ & $0.611 \pm 0.041(6.8)$ \\
\hline $\mathrm{C}_{18} \mathrm{Hydra}_{1}$ & $0.204 \pm 0.028(13.6)$ & $0.673 \pm 0.072(10.7)$ \\
\hline $\mathrm{C}_{18}$ ec & $0.209 \pm 0.048(22.9)$ & $0.576 \pm 0.107(18.6)$ \\
\hline $\mathrm{C}_{6} \mathrm{H}_{11}$ ec & $0.160 \pm 0.013(8.0)$ & $0.649 \pm 0.043(6.6)$ \\
\hline $\mathrm{C}_{6} \mathrm{H}_{5}$ & $0.255 \pm 0.083(32.5)$ & $0.572 \pm 0.054(9.5)$ \\
\hline $\mathrm{CN}$ & $0.134 \pm 0.007(5.2)$ & $0.554 \pm 0.023(4.1)$ \\
\hline $\mathrm{NH}_{2} / \mathrm{C}_{18}$ & $0.223 \pm 0.030(13.4)$ & $0.614 \pm 0.065(10.6)$ \\
\hline $\mathrm{OH}$ & $<\mathrm{LOQ}$ & $0.067 \pm 0.006(8.8)$ \\
\hline $\mathrm{NO}_{2}$ & $0.133 \pm 0.007(5.4)$ & $0.557 \pm 0.025(4.5)$ \\
\hline Easy & $0.199 \pm 0.028(14.3)$ & $0.553 \pm 0.068(12.3)$ \\
\hline $\mathrm{HR}-\mathrm{P}$ & $0.184 \pm 0.022(11.7)$ & $0.520 \pm 0.046(8.8)$ \\
\hline $\mathrm{PA}$ & $<\mathrm{LOQ}$ & $0.075 \pm 0.006(7.9)$ \\
\hline Strata-X & $0.284 \pm 0.042(14.9)$ & $0.963 \pm 0.034(3.5)$ \\
\hline $\mathrm{Cellulose}$ & $<\mathrm{LOQ}$ & $<\mathrm{LOQ}$ \\
\hline Davisil & $<\mathrm{LOQ}$ & $<\mathrm{LOQ}$ \\
\hline Florisil & $<\mathrm{LOQ}$ & $0.046 \pm 0.011(23.9)$ \\
\hline
\end{tabular}

In bath additive 2, menthol and p-cymene were determined. For menthol Strata-X followed by $\mathrm{C}_{18}$ Hydra gained highest amounts. Strata- $\mathrm{X}, \mathrm{C}_{8}$ and $\mathrm{C}_{4}$ had comparable results in case of p-cymene. Surprisingly Normal Phases like Davisil, $\mathrm{CN}$, and $\mathrm{NO}_{2}$ showed good results, probably caused by tensides in the matrix.

\subsubsection{Cough Drops}

In the cough drops, menthol was quantified (Table 10). Highest amounts were found with Strata-X, $\mathrm{C}_{18}$ Hydra and $\mathrm{C}_{18}$ ec.

\section{CONCLUSION}

These experiments clearly show the necessity to test a broad range of available SPE materials for method development, as the matrix has great influence on recovery rates of different analytes. Automation is a useful tool to 
Table 10. Concentration of Menthol in $\mathrm{mgdrop}^{-1}$ After Automated Extraction of Cough Drops. Also Given are Standard Deviation and Coefficient of Variation

\begin{tabular}{|c|c|}
\hline & Menthol \\
\hline $\mathrm{C}_{2}$ & $0.082 \pm 0.016(19.0)$ \\
\hline $\mathrm{C}_{4}$ & $0.529 \pm 0.087(16.4)$ \\
\hline $\mathrm{C}_{8}$ & $1.724 \pm 0.312(18.1)$ \\
\hline $\mathrm{C}_{18}$ Hydra & $2.241 \pm 0.263(11.7)$ \\
\hline $\mathrm{C}_{18} \mathrm{ec}$ & $2.375 \pm 0.261(11.0)$ \\
\hline $\mathrm{C}_{6} \mathrm{H}_{11} \mathrm{ec}$ & $0.585 \pm 0.162(27.7)$ \\
\hline $\mathrm{C}_{6} \mathrm{H}_{5}$ & $1.399 \pm 0.315(22.5)$ \\
\hline $\mathrm{CN}$ & $1.807 \pm 0.741(41.0)$ \\
\hline $\mathrm{NH}_{2} / \mathrm{C}_{18}$ & $<$ LOQ \\
\hline $\mathrm{OH}$ & $0.056 \pm 0.024$ \\
\hline $\mathrm{NO}_{2}$ & $0.208 \pm 0.030(14.2)$ \\
\hline Easy & $0.844 \pm 0.100(11.9)$ \\
\hline HR-P & $0.798 \pm 0.039(4.9)$ \\
\hline PA & $0.090 \pm 0.009(9.6)$ \\
\hline Strata-X & $2.404 \pm 0.190(7.9)$ \\
\hline Cellulose & $<$ LOQ \\
\hline Davisil & $<\mathrm{LOQ}$ \\
\hline Florisil & $0.075 \pm 0.007(8.8)$ \\
\hline
\end{tabular}

accomplish the emerging high number of samples. Fully automated extraction procedure with the MEA Personal Purification System ${ }^{\circledR}$ took 5 h 15 min for 12 samples. Preparing the system takes about $10 \mathrm{~min}$, extraction of analytes about 15 $\mathrm{min}$. So laboratory staff is occupied for $25 \mathrm{~min}$, while the rest is done by the robot. To do the same extraction manually one would have to invest about 4 hours using a syringe and adapter and about 2 hours using a vacuum manifold. Apart from time, another advantage of automation is the fact, that mistakes made by laboratory staff are minimized. They will not be exhausted due to the long extraction procedure and there will not be deviations due to the fact that different laboratory workers are doing the extractions.

In many cases, the most commonly used SPE material $\mathrm{C}_{18}$ showed lower recovery rates compared to Strata-X or $\mathrm{C}_{8}$. Strata$\mathrm{X}$ was in 12 cases the best material. Only for the extraction of menthol out of cough drops, $\mathrm{C}_{18}$ ec and $\mathrm{C}_{18}$ Hydra obtained equal results. In case of the extraction of p-cymene out of bath additive 2 nearly equal results were obtained with $\mathrm{C}_{8}$ and $\mathrm{C}_{4}$.

Furthermore results show, that modern SPE materials like Strata-X can be better used in automated extraction procedures than the "old schooled" ones like silica based materials.

\section{CONFLICT OF INTEREST}

The authors confirm that this article content has no conflict of interest.

\section{ACKNOWLEDGEMENTS}

The authors want to thank Eurasia-Pacific Uninet (EPU) (Salzburg, Austria), the Ministry for Science and Research and the Ministry for Health, Family and Youth (Vienna, Austria) (Novel Analytical Tools for Quality Control in Traditional Chinese Medicine, Project No. 80855).

\section{REFERENCES}

[1] Fritz, J.S. Analytical Solid Phase Extraction. Wiley-VCH: New York, Chichester, Weinheim, Brisbane, Singapore, Toronto, 1999.

[2] Hennion, M.-C. Solid-phase extraction: method development, sorbents, and coupling with liquid chromatography. J. Chromatogr. A, 1999, 856, 3-54.

[3] Poole, C.F. New trends in solid-phase extraction. Trends Anal. Chem. 2003, 22, 362-373.

[4] Stecher, G.; Jarukamjorn, K.; Zaborski, P.; Bakry, R.; Huck, C.W.; Bonn, G.K. Evaluation of extraction methods for the simultaneous analysis of simple and macrocyclic trichothecenes. Talanta, 2007, 73, 251-257.

[5] Lehotay, S.J. Quick, easy, cheap, effective, rugged, and safe approach for determining pesticide residues. Pestic. Protoc., 2006, 239-261.

[6] Huck, C.W.; Bonn, G.K. Recent developments in polymer based sorbents for solid-phase extraction. J. Chromatogr. A 2000, 885, 51-72.

[7] King, J.N.; Fritz, J.S. Concentration of metal ions by complexation with sodium bis(2-hydroxyethyl)dithiocarbamate and sorption on XAD-4 resin.Anal. Chem. 1985, 57,1016-1020.

[8] Sun, J.J.; Fritz, J.S. Chemically modified polymeric resins for highperformance liquid chromatography. J. Chromatogr. A, 1990, 522, 95105.

[9] Sun, J.J.; Fritz, J.S. Chemically modified resins for solid-phase extraction. J. Chromatogr. A, 1992, 590, 197-202.

[10] Marvin, C.H.; Brindle, I.D.; Hall, C.D.; Chiba, M. Automated highperformance liquid chromatography for the determination of pesticides in water using solid phase extraction. Anal. Chem., 1990, 62, 14951498.

[11] Junk, G.A.; Richard, J.J. Organics in water: solid phase extraction on a small scale. Anal. Chem., 2002, 60, 451-454.

[12] Junk, G.A.; Richard, J.J.; Grieser, M.D.; Witiak, D.; Witiak, J.L; Arguello, M.D.; Vick, R.; Svec, H.J.; Fritz, J.S.; Calder,G.V. Use of macroreticular resins in the analysis of water for trace organic contaminants. J. Chromatogr. A, 1974, 99, 745-762.

[13] Krieg, C. New analytical tools in the field of extra virgin olive oil, in Institut für Analytische Chemie und Radiochemie. Leopold-Franzens Universität: Innsbruck. p. 254, 2010.

[14] Feuerstein, I.; Bonn, G.; Gjerde, D.; Huck, C.; Stecher, G. Automated method and device for preparing an analyte for analysis by MALDI mass spectrometry using columns in combination with a liquid handling system. PCT Int. Appl. 52pp. CODEN: PIXXD2 WO 2008045526 A2 20080417 CAN 148:444249 AN 2008:4744512008, 2008

[15] Jarrett, D. Improved Clean Up and Recovery of Pharmaceutical Compounds From Plasma using Strata ${ }^{\mathrm{TM}}-\mathrm{X}$ Solid Phase Extraction (SPE) vs Traditional Liquid-Liquid Extraction Methods. Available at: https://phenomenex.blob.core.windows.net/documents/7138ac21 -4b b9-4204-9e1e-b17df4ff957e.pdf [accessed 2011].

[16] Wissiack, R.; Rosenberg, E.; Grasserbauer, M. Comparison of different sorbent materials for on-line solid-phase extraction with liquid chromatography-atmospheric pressure chemical ionization mass spectrometry of phenols. J. Chromatogr. A, 2000, 896, 159-170. 